\title{
Use of Proplast II as a subperiosteal implant for the correction of anophthalmic enophthalmos
}

\author{
S Shah, M Rhatigan, R Sampath, C Yeoman, S Sunderland, R Brammer, \\ B Leatherbarrow
}

\begin{abstract}
Background-A variety of autogenous and alloplastic materials have been used as subperiosteal implants to correct anophthalmic enophthalmos. Proplast II is a synthetic porous composite of Teflon polymer and alumina. Proplast II offers a number of advantages over other commonly used alloplastic materials such as silicone and polymethyl methacrylate. It is light, porous, resilient, malleable, and easy to shape. It can be readily sterilised after shaping. It has been found to integrate with the surrounding tissues, thereby minimising the risk of subsequent implant migration and extrusion.

Methods-Proplast II was used as a subperiosteal implant in a total of 15 anophthalmic patients during the period June 1990 to March 1994. The indication for this procedure in all patients was poor orbital volume replacement despite the prior insertion of an adequately sized spherical socket implant.

Results-The results were excellent with a good correction of preoperative upper eyelid sulcus deformity. There were no operative complications nor any serious postoperative complications. The implants were well tolerated.
\end{abstract}

Conclusion-Proplast II can be highly recommended for use as a subperiosteal implant.

(Br f Ophthalmol 1995; 79: 830-833)

Anophthalmic patients are often found to be volume deficient even after placement of an adequately sized orbital implant. This can lead to poor cosmesis owing to the development of a deep upper lid sulcus deformity or upper lid retraction and enophthalmos (the post-enucleation socket syndrome). Various materials have been used to replace this lost volume (inserted into different orbital spaces) for example, autologous or homologous bone, ${ }^{1}$ cartilage, ${ }^{2}$ dermal grafts, ${ }^{1}$ glass beads, ${ }^{3}$ plastic plate, ${ }^{4}$ and more recently silicone, ${ }^{5-7}$ and Proplast I. ${ }^{8}$

We describe the use of Proplast II for the correction of this upper lid sulcus deformity and enophthalmos with a preshaped implant inserted into the inferior subperiosteal space via a lateral canthal approach. ${ }^{79}$

Patients and methods

During the period June 1990 to March 1994, 15 anophthalmic patients underwent surgery consisting of implantation of Proplast II into the subperiosteal space of the floor of the orbit. The patients were selected for surgery on the basis of poor cosmesis owing to the presence of a deep upper lid sulcus and enophthalmos despite the presence of an adequately sized spherical socket implant. All the patients were seeking an improvement in their cosmetic appearance.

Nine patients were male and six female. The age range was 27 to 66 years with a mean of 46 years. Six of the patients had undergone an enucleation with placement of a primary implant. The other nine patients had previously undergone a simple enucleation with the later placement of a secondary implant. Of these implants, 10 were baseball implants and five hydroxyapatite implants. The condition leading to enucleation was trauma in 11 cases, choroidal melanoma in one case, and congenital glaucoma with the development of a blind painful eye in three cases. There were no preoperative complicating factors. None of the patients had a missed orbital fracture as the cause of the residual volume deficit. None of the patients had a contracted socket. The surgery was performed under general anaesthesia in all cases.

\section{Surgical technique}

The Proplast implant is fashioned by the ocularist, before surgery. A wax mould of an orbital floor is prepared using an adult male skull with average orbital dimensions. The mould is used as a template for the implant which is carved from a Proplast II block, the dimensions of each block being $60 \times 40 \times 10$ $\mathrm{mm}$. Each block will provide two implants. The block is carved using a scalpel in a clean field. As this material is easy to carve, it can be shaped to give maximum thickness posteriorly while allowing the edge which lies along the inferior orbital rim to be less than $1 \mathrm{~mm}$ in thickness. This obviates the need for removal and debulking of the implant, and because of the material's low modulus of elasticity this edge can be moulded slightly around the rim without any springing. When finished the Proplast implant weighs about one third of a similarly sized silicone implant. Steam sterilisation is performed in a standard gravity steriliser for 30 minutes at $121^{\circ} \mathrm{C}$ and $1.5 \mathrm{~kg} / \mathrm{cm}^{2}$. It is important that at no time should ungloved hands come into contact with the implant, as it will absorb the skin's natural oils rendering the implant useless.

A skin incision is marked beginning at the lateral canthus and extending laterally and 


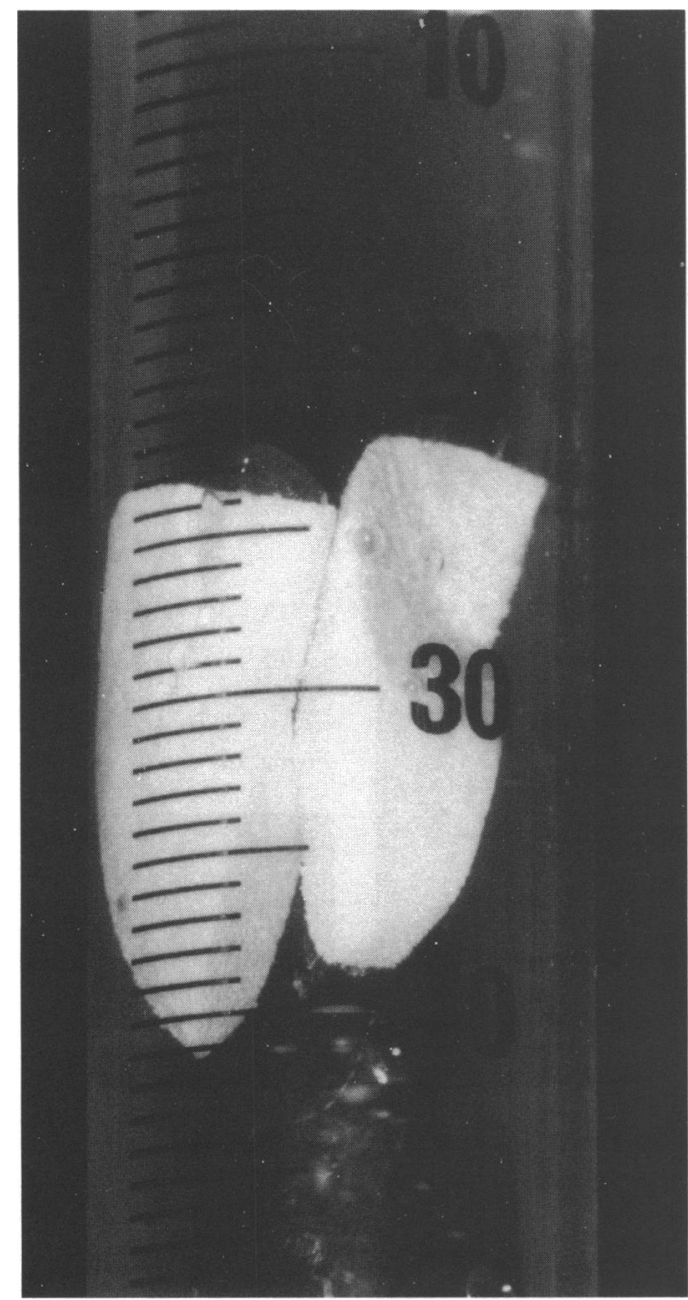

Figure 1 Proplast II within syringe, impregnated with gentamicin.

slightly downwards for a distance of approximately $2.5 \mathrm{~cm}$ (a similar approach to that used by Rose et $a l^{7}$ ).

A skin incision is made using a number 15 Baird-Parker blade along the line marked.... The underlying orbicularis oculi muscle is bluntly dissected in a vertical fashion using Steven's scissors down to the lateral orbital rim which is then exposed using a Freer periosteal elevator. The periosteum of the lateral orbital rim is incised vertically $3 \mathrm{~mm}$ beyond the lateral orbital rim and the periosteum is elevated from the underlying bone using the

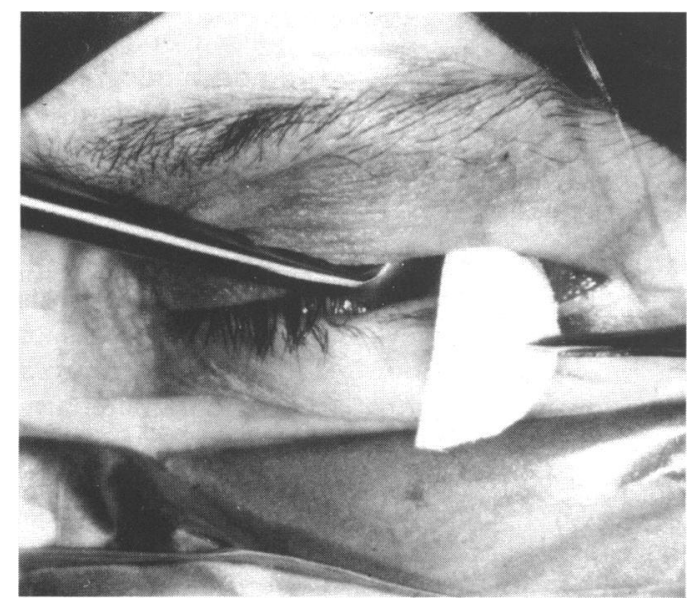

Figure 2 Insertion of Proplast II into subperiosteal space.
Freer periosteal elevator. The periosteum is elevated from the inferolateral orbital wall and the orbital floor. The zygomaticofacial artery and vein are identified and cauterised. Once the space created is large enough to accommodate the implant, the implant is divided in two lengthways using a number 15 Baird-Parker blade. The implant is impregnated with a gentamicin and saline solution $(80 \mathrm{mg}$ in $50 \mathrm{ml}$ ) by inserting the two pieces into a $50 \mathrm{ml}$ syringe and alternately depressing and withdrawing the plunger, expelling the air from implant (Fig 1). The two pieces are then placed into the subperiosteal space to lie side by side on the orbital floor (Fig 2). A no touch technique is observed during manipulation of the implant. Once the position of the implant is satisfactory, the periosteum is closed using interrupted 5/0 Vicryl sutures on a half circle needle. If the lower lid has excessive laxity, a lateral tarsal strip procedure ${ }^{10}$ can be performed at this stage. The muscle layer is closed using the same suture. The skin edges are reapproximated using interrupted $7 / 0$ Vicryl sutures. Topical antibiotic ointment is applied to the wound and the patient receives a 1 week course of systemic antibiotics.

\section{Results}

All 15 patients tolerated the procedure well with no intraoperative complications. There were no immediate postoperative complications, in particular, orbital haemorrhage and infection did not occur. Postoperative pain was easily controlled with simple analgesics only. During the follow up period (ranging from 3 months to 4 years), two minor postoperative complications were noted. One patient developed a wound inclusion cyst some months after surgery which was excised without further problem. One patient continues to have mild infraorbital hypoaesthesia at 3 months' follow up. In no case did extrusion or migration of the implant occur. Three patients required a lateral tarsal strip procedure to correct lower lid laxity. In six of the other patients this had been performed at the time of implantation. One further patient required a levator aponeurosis advancement. All patients had a marked increase in orbital volume, as shown by the improvement in the degree of enophthalmos, the loss of the upper lid sulcus deformity or upper lid retraction, and by the reduction in size of the required prosthesis (Figs $3 \mathrm{~A}$ and $3 \mathrm{~B}$, $4 \mathrm{~A}$ and $4 \mathrm{~B}$ ). All 15 patients were pleased with the improvement in their cosmetic appearance.

\section{Discussion}

Proplast was originally used as a composite of polytetrafluoroethylene (PTFE) and vitreous carbon fibres (Proplast I) by Homsy et al ${ }^{11}$ in 1970. It has been used extensively ${ }^{12}$ for 20 years in orthopaedic, ${ }^{13}$ otolaryngotic, ${ }^{14}$ and oral surgery. ${ }^{15}$ It was described in ophthalmology by Lyall ${ }^{16}$ in 1976 (as orbital implants following enucleation) and Lamberts and Grandon $^{17}$ in 1978 (as a keratoprosthesis). It was used by Bello and Levine ${ }^{18}$ in 1980 (direct 


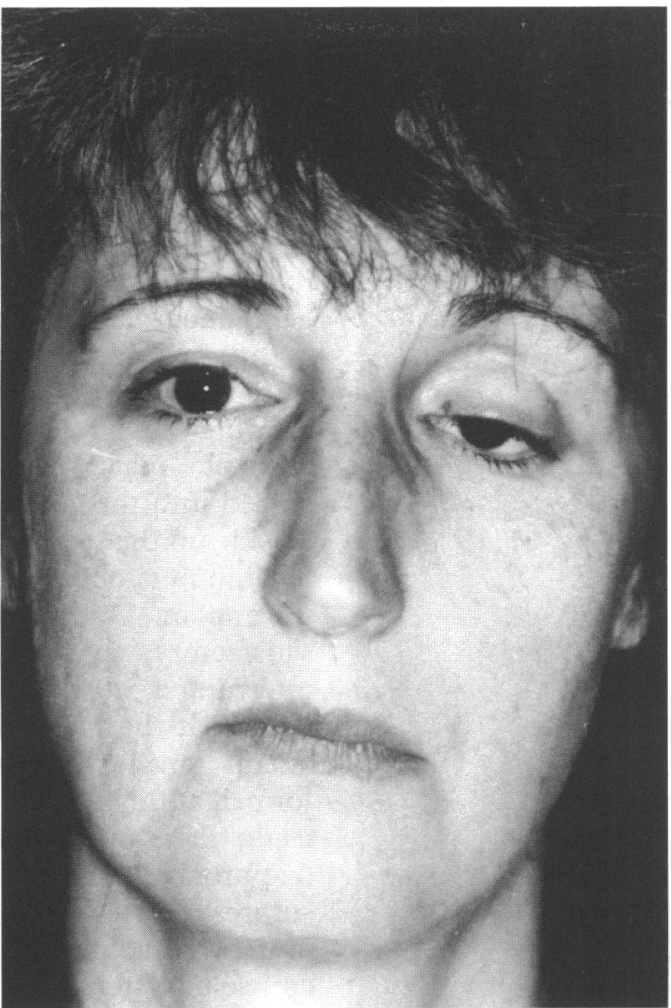

Figure $3 A$

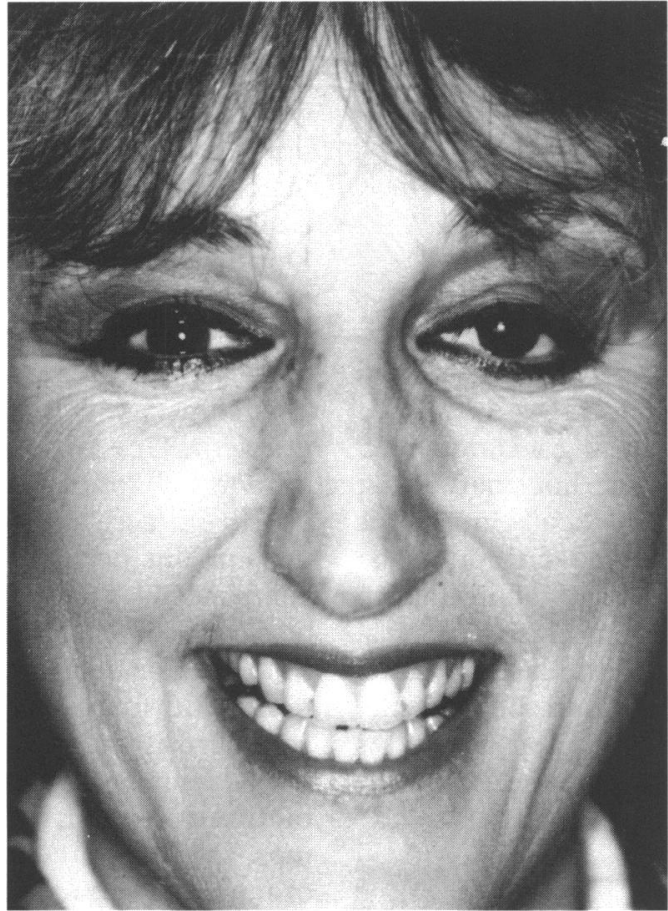

Figure 3B

Figure 3 Example 1: appearance before $(A)$ and after (B) surgery.

insertion of Proplast I into the superior sulcus) and $\mathrm{Ma}$ et al ${ }^{19}$ in 1987 (subperiosteally via an infraciliary approach in addition to a SpiveyAllen acrylic floor implant in two out of four cases) for correction of superior sulcus deformities. To our knowledge, this is the first description of Proplast II being used to correct the superior sulcus deformity of the anophthalmic socket via a lateral canthal approach. Proplast II is a white composite (Proplast I was grey) of PTFE and alumina. Physically it is

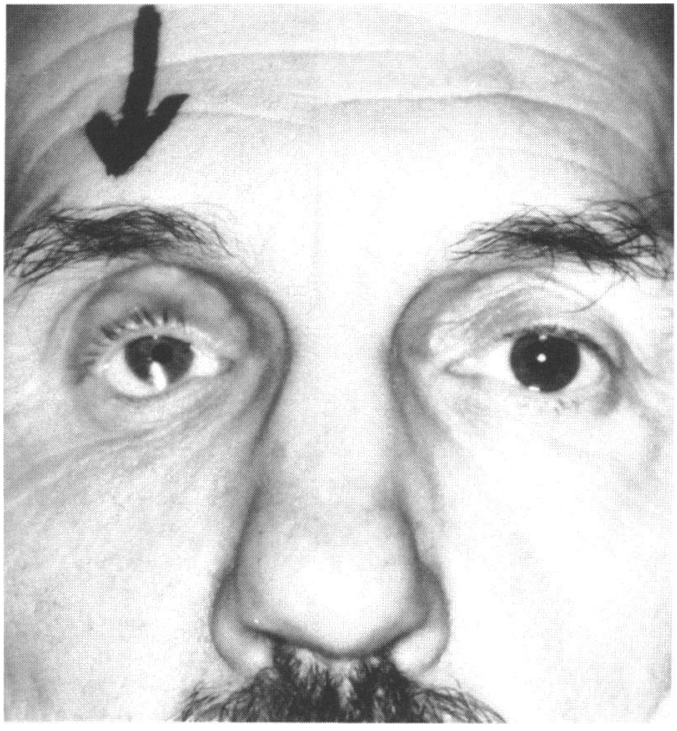

Figure $4 A$

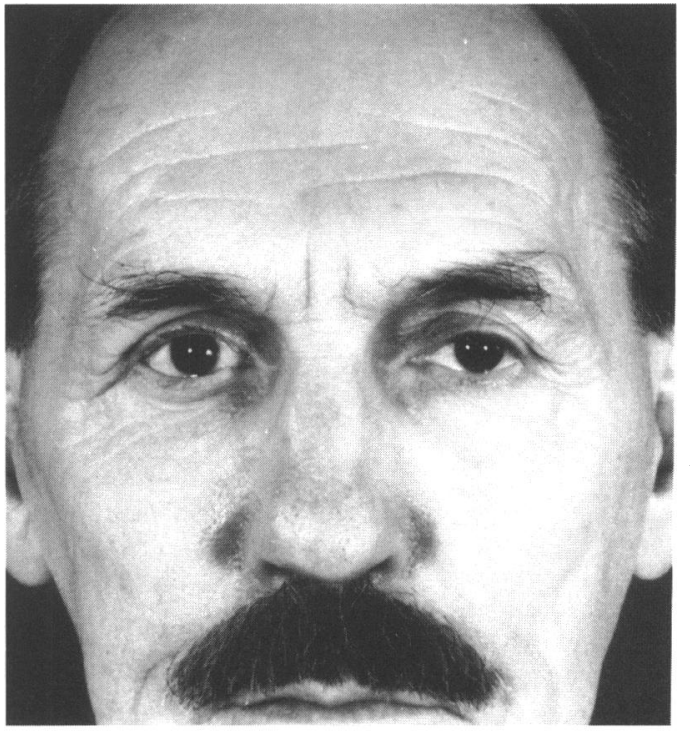

Figure $4 B$

Figure 4 Example 2: appearance before $(A)$ and after (B) surgery.

very light with a porosity of $70-90 \%$ of the volume of the material. The pore size varies from $50-400 \mu \mathrm{m} .{ }^{20}$ It is malleable, easy to handle, and it can be shaped with scissors or a knife. It is well tolerated, and integrates with the surrounding tissues by fibrous ingrowth. Thus it is very stable with a very low risk of migration or extrusion. It is inert, non-toxic, and non-biodegradable.

Orbital implants do not replace all of the orbital volume deficiency following enucleation. A $14 \mathrm{~mm}$ diameter sphere replaces 1.4 $\mathrm{ml}$, and $18 \mathrm{~mm}$ sphere replaces $3.0 \mathrm{ml}$, and a $20 \mathrm{~mm}$ sphere replaces $4 \cdot 2 \mathrm{ml}$. The total volume loss depends on the volume of the globe and any associated orbital fat atrophy. This averages approximately $7 \cdot 0 \mathrm{ml}$.

Previous materials used for management of the superior sulcus deformity of the anophthalmic socket have to be harvested if they are autologous or homologous and do not have a completely predictable effect on orbital volume. Other material such as glass beads and plastic plates are likely to be extruded. The advantages 
and disadvantages of silicone have been reported extensively. ${ }^{72122}$ Silicone does not stabilise because tissue ingrowth cannot occur. The implant moves with even the slightest pressure; this movement can result in extrusion of the implant. Proplast I had been shown to cause secondary conjunctival melanosis ${ }^{23}$ and, as it is grey, may become visible through thinned tissues. It has been superseded by Proplast II. ${ }^{24}$ We believe Proplast II offers a number of significant advantages over other available materials for the management of a deep upper lid sulcus deformity and is extremely well tolerated without the risk of extrusion.

1 DeVoe AG. Experiences with the surgery of the anophhalmic orbit. Am $\mathcal{F}$ Ophthalmol 1945; 28: 1346-51.

2 Zbylski JK. Correction of lower eyelid ptosis in the anophthalmic socket with an autogenous ear cartilage graft. Plast Reconstr Surg 1977; 61: 220-3.

3 Smith B, Obear M, Leone CR. The correction of enophthalmos associated with anophthalmos by glass bead implantation. Am f Ophthalmol 1967; 64: 1088-93.

4 Sugar HS, Forrester HJ. Methacrylic resin implants for sunken upper eyelid following enucleation. $A m f$ sunken upper eyelid following

5 Vistnes IM, Paris GL. Uses of RTV silicone in orbital reconstruction. Am $f$ Ophthalmol 1976; 83: 577-81.

6 White RH, Shannon GM, Yassin J. A modification of the correction of enophthalmos. Arch Ophthalmol 1972; 87: 652-4

7 Rose GE, Sigurdsson H, Collin R. The volume-deficient orbit: clinical characteristics, surgical management, and results after extraperiorbital implantation of Silastic block. Br ₹ Ophthalmol 1990; 74: 545-50.

8 Collett R, Garber P, Sachs SA. The surgical management of the anophthalmic syndrome with the concomitant malunion of the zygomatico-maxillary complex. F Oral Maxillofac Surg 1987; 45: 341-5.

9 Collin JRO. Lateral canthal approach to orbital floor: a manual of systemic eyelid surgery. Edinburgh: Churchill Livingstone, 1989: 136

10 Anderson RL, Gordy DD. The tarsal strip procedure. Arch Ophthalmol 1979; 97: 2192-6.

11 Homsy CA, King JW, Cain TE, Anderson MS. Dynamic stabilization of implanted prostheses. $₹$ Bone foint Surg stabilization of

12 Freeman BS, Wiener DR. Clinical uses of Proplast: expectations and results. In: Rubin A, ed. Biomaterials in reconstructive surgery. St Louis: Mosby, 1983; Ch 31: 494-508.

13 Corn RC, Berry JL, Black JD, Greenwald AS. Mechanical and microangiographic analysis of porous implant systems. Transactions, 24th annual meeting of the Orthopaedic Research Society. Dallas, Texas, 1978: 161

14 Shea JJ, Homsy CA. The uses of Proplast in otologic surgery. Laryngoscope $1974 ; 84$ : 1835-45.

15 Hinds EC, Homsy CA, Kent N . Use of a biocompatible interface for binding tissues and prostheses in temperomandibular joint surgery. Oral Surg, Oral Med Oral Path mandibular joint

16 Lyall MG. Proplast implant in Tenon's capsule after excision of the eye. Trans Ophthalmol Soc UK 1976; 96: 79-81.

17 Lamberts DW, Grandon SC. A new alloplastic material for ophthalmic surgery. Ophthalmic Surg 1978; 9: 35-42.

18 Bello VM, Levine MR. Superior sulcus deformity. Arch Ophthalmol 1980; 98: 2215-6.

$19 \mathrm{Ma} \mathrm{L}$, Wong SP, Wu CY, Yao SJ. Orbital reconstruction with proplast. Ophthalmic Plast Reconstr Surg 1987; 3: 151-7.

20 Product information sheet Novamed Inc, Houston, Texas.

21 Motou Y. The complications of augmentation rhinoplasty in orientals. Br F Plast Surg 1980; 4: 79.

22 Shirakabe Y, Shirakabe T, Takayanagi S. A new type of prostheses for augmentation rhinoplasty: our experience in 1600 cases. Br $\mathcal{F}$ Plast Surg 1981; 34: 353.

23 Skouteris CA, Soutereanos GC. Secondary acquired melanosis resulting from an alloplastic implant. $\mathcal{f}$ Oral Maxillofac Surg 1988; 46: 603-5.

24 Westfall RL A comparison of porous composite PTFE/graphite and PTFE/alumina facial implants in primates. F Oral Maxillofac Surg 1982; 40: 771-5. 